\title{
Frequency and Antibiotic Resistance Pattern of Diarrheagenic Escherichia coli (DEC) Strains Isolated from Children Aged Less Than 10 Years
}

\author{
Shahram Shahraki Zahedani (PhD) \\ Department of Microbiology, School of \\ Medicine, Infectious Diseases and \\ Tropical Medicine Research Center, \\ Zahedan University of Medical \\ Sciences, Zahedan, Iran \\ Nasrin Sayadzai (BSc) \\ Department of Microbiology, Faculty \\ of Medicine, Zahedan University of \\ Medical Sciences, Zahedan, Iran \\ Corresponding author: Nasrin \\ Sayadzai \\ Tel: +985413414558 \\ E-mail: Nasrinsayadzai@gmail.com \\ Address: Zahedan University of \\ Medical Sciences, Zahedan, Iran \\ Received : 08 Jun 2017 \\ Revised: 01 Aug 2017 \\ Accepted: 26 Aug 2017
}

Shahram Shahraki Zahedani https://orcid.org/00000003-4348-8184

\section{ABSTRACT}

Background and Objectives: Diarrheagenic Escherichia coli (DEC) pathotypes are important causes of diarrhea among children in developing countries. The objective of this study was to determine the frequency and antibiotic resistance pattern of DEC pathotypes in children aged less than 10 years.

Methods: This cross-sectional study was done on $300 \mathrm{E}$. coli strains isolated from diarrheic stool samples of children aged less than 10 years who were admitted to hospitals and central laboratory in Zahedan, between July and 0ctober 2016. DEC pathotypes were identified by standard biochemical testing and phenotypic testing using polyvalent antiserums. Antibiotic resistant pattern of these strains was evaluated against 11 different antibiotics by the agar disk diffusion method according to the Clinical and Laboratory Standards Institute guidelines.

Results: of the 300 E. coli isolates, 89 (29.6\%) were found positive for DEC using polyvalent antiserums. In this study, 35 cases (39.3\%) reacted with antiserum 1, 21 cases $(25.8 \%)$ reacted with the antiserum 2 , and 31 cases $(34.8 \%)$ reacted with antiserum 3 . The highest rate of resistance was observed against ampicillin $(94.8 \%)$, tetracycline $(87.2 \%)$, and co-trimoxazole (70.5\%). In addition, the lowest rate of resistance was related to imipenem $(1 \%)$ and ciprofloxacin $(8.9 \%)$.

Conclusion: IEC pathotypes are the important causes of diarrhea among children admitted to hospitals of Zahedan. Considering the high rate of antibiotic resistance among these pathotypes in this region, prescription of antibiotics should be based on accurate detection of these strains.

Keywords: Escherichia coli, Child, Antibiotic Resistance. 


\section{INTRODUCTION}

Diarrhea is one of the causes of children mortality in developing countries. According to the World Health Organization, diarrhea accounts for approximately 2.5 million child deaths worldwide. Diarrhea results in severe dehydration and malnutrition in children, and can lead to death if not diagnosed and treated early. Various microorganisms can cause diarrhea, but the most important cause of this condition is Escherichia coli, which is often associated with mortality and increased medical costs (1). Diarrheagenic Escherichia coli (DEC) pathotypes are the most common bacterial causes of diarrhea in children (2). Based on their virulence properties and mechanisms, and clinical signs, DEC strains are divided into six main pathotypes including Enterotoxigenic E. coli (ETEC), Enteropathogenic E. coli (EPEC), Enteroinvasive E. coli, Shiga toxin-producing E. coli, Enteroaggregative E. coli, and diffuse adhering E. coli. Each DEC pathotype has unique pathogenic properties, making them important from the medical aspect (3). Since $E$. coli is part of the normal intestinal microflora of humans, identifying diarrheacausing strains requires phenotypic and genotypic testing based on the pathogenic indices. Polyvalent antiserums can be used for the phenotypic detection of these strains. The technique is based on identification of $E$. coli somatic (O) and flagellar (H) antigens using commercial antiserums of $\mathrm{O}$ and $\mathrm{H}$. In addition, multiplex-PCR (M-PCR) could be used as molecular technique for the detection of $E$. coli pathotypes (4).

Antimicrobial resistance among $E$. coli strains has been reported worldwide. Moreover, treatment of $E$. coli infections has become more complicated due to the emergence of strains resistant to most first-line antimicrobial agents (5). Antibiotic resistance among DEC pathotypes is of great importance because of the high incidence rate in children (6). Given the important role of E.coli pathotypes in causing diarrhea and lack of robust studies on these strains in developing countries such as Iran, detection of these strains and evaluation of their antibiotic resistance pattern seem necessary. Therefore, this study was conducted to resolve the defects in the current methods of detecting DEC pathotypes, to evaluate the prevalence and pattern of antibiotic resistance among the strains isolated from children with diarrhea.

\section{MATERIAL AND METHODS}

This cross-sectional study was conducted between July and October 2016. Stool samples were collected from diarrheic children aged less than ten years who were admitted to the hospitals and central laboratory of Zahedan, Iran. The samples were examined a few hours after the collection. The samples were first cultured on different media including MacConkey, blood, eosin-methylene blue, and xylose lysine deoxycholate agar. After 24 hours of incubation, culture plates were examined for the presence of $E$. coli. Identity of suspected $E$. coli colonies was evaluated using biochemical tests including triple sugar iron agar, sulfur indole motility, methyl red/Voges-Proskauer, Simmons citrate agar, urease, and lysine decarboxylase (all media were purchased from Merck, Germany). E. coli pathotypes were detected using polyvalent antiserums (Sifin, Germany) through slide agglutination test. (In this method, several purified colonies from 18-hour culture of bacteria in TSI were mixed with a drop of antiserums provided in the kit.Agglutination of antiserum drop after 40 seconds indicated a positive reaction. Kirby-Bauer antibiotic testing was performed on Mueller-Hinton agar according to the Clinical and Laboratory Standards Institute's (CLSI) guidelines. Commercial dehydrated antibiotic discs (Mast, UK) used in the study were as follows: tetracycline $(30 \mu \mathrm{g})$, chloramphenicol $(30 \mu \mathrm{g})$, co-trimoxazole $(25 \mu \mathrm{g})$, cefixime $(5 \mu \mathrm{g})$, gentamicin $(10 \mu \mathrm{g})$, ciprofloxacin $(5 \mu \mathrm{g})$, imipenem $(15 \mu \mathrm{g})$, cefotaxime $(30 \mu \mathrm{g})$, ceftriaxone $(30 \mu \mathrm{g})$, nalidixic acid $(30 \mu \mathrm{g})$, and ampicillin $(10 \mu \mathrm{g})$. Bacterial concentration equivalent to $0.5 \mathrm{McFarland}$ standard was cultured on Mueller Hinton agar. After placing the antibiotic disks on the medium, the plate was incubated at $37{ }^{\circ} \mathrm{C}$ for $18-24$ hours. Diameter of inhibition zone around the discs was measured in millimeters. The isolates resistant or susceptible to the antibiotics were characterized according to the manufacturer's instructions (7). In addition, E. coli ATCC 25922 was used as control in the antibiogram. 


\section{RESULTS}

Among the 300 E. coli samples, 173 were from males and 127 from females. In addition, 195 samples were collected from inpatients and 105 from outpatients. Polyvalent antiserums in the kit are placed in 3 Serogroups (Table 1). Among the samples found positive for DEC, 31 samples reacted with antiserum 1, 35 samples reacted with antiserum 2, and 23 samples reacted with antiserum 3 (Table 2). Overall, 89 E. coli strains $(29.6 \%)$ were positive for DEC when using the polyvalent antiserums.

The DEC serotypes identified in the previous experiment were evaluated for antibiotic resistance.

The highest rate of antibiotic resistance was observed against ampicillin, tetracycline, and co-trimoxazole. The lowest rate of antibiotic resistance was related to imipenem and ciprofloxacin (Figure 1).

Table 1- Serotypes of $E$. coli that could be identified by the commercial kit used in this study

\begin{tabular}{ccc}
\hline Antiserum & \multicolumn{1}{c}{ Identifiable serotypes } \\
\hline Anti-coli 1 & O26:K60 & O44:K74 O114:K90 O125:K70 O142:K86 O158:K- \\
Anti-coli 2 & O55:K59 O86:K61 O91:K- O111:K58 O119:K69 O126:K71 O127:K63 O128:K67 \\
& \\
Anti-coli 3 & O25:K11 O78:K80 O103:K- O118:K- O124:K72 O145:K- O157:K- O164:K-
\end{tabular}

Table 2- Number and percentage of DEC strains identified by the polyvalent antiserums

\begin{tabular}{|c|c|c|}
\hline Antiserum & $\mathbf{N}$ & $\%$ \\
\hline Antiserum 1 & 31 & 34.4 \\
\hline Antiserum 2 & 35 & 39.3 \\
\hline Antiserum 3 & 23 & 8 \\
\hline
\end{tabular}

Figure 1- Percentage of antibiotic resistance of DEC isolates from diarrheic children aged less than 10 years

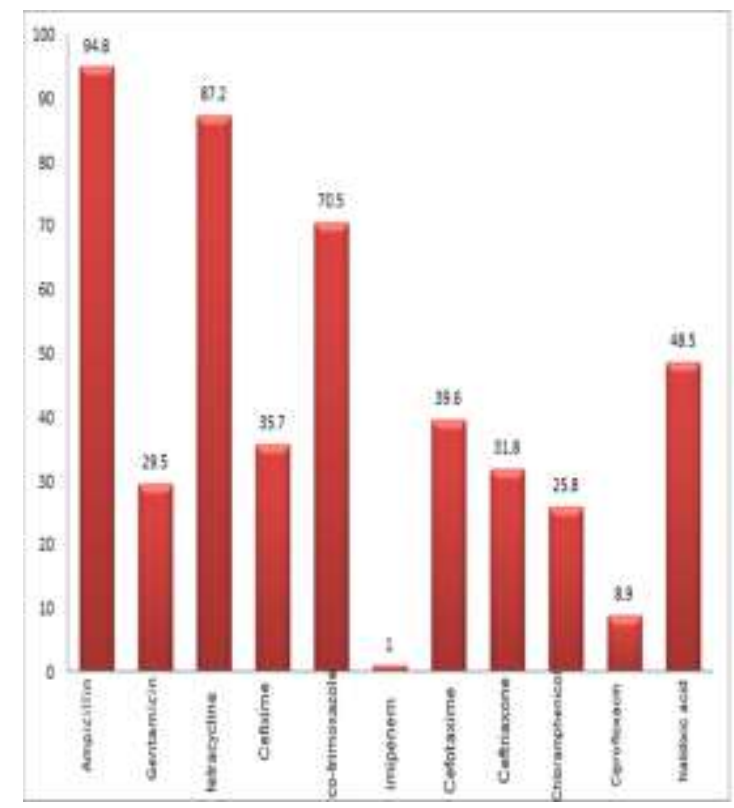




\section{DISCUSSION}

Various bacteria, viruses and unicellular microorganisms can cause diarrhea. Salmonella, Shigella, Yersinia enterocolitica, Vibrio, Campylobacter, and various pathotypes of E. coli are among the bacterial causes of diarrhea (8). DEC pathotypes are important pathogens and one of the common causes of mortality among children, particularly in developing countries (9). Prevalence of these pathotypes depends on the geographical area, health status, and socioeconomic conditions. DEC pathotypes are accountable for approximately $30-40 \%$ of diarrhea cases, $15-30 \%$ of which require hospital care $(10,11)$. In this study, we evaluated the prevalence of DEC pathotypes and their antibiotic susceptibility patterns among strains isolated from children with diarrhea in Zahedan, Iran. We found that 89 of 300 samples $(29.6 \%)$ were positive for DEC. Identification of new strains is of great importance due to the emergence of new pathogenic serotypes and consequently the increasing resistance of these strains to antibiotics, as well as the lack of common diagnostic techniques for accurate detection of these strains(12).

Since E.coli strains are part of the intestinal microflora, the identification of DEC strains requires phenotypic and genotypic assessment based on the pathogenic indices [13]. The results of the present study also showed the high prevalence of DEC strains in diarrheic children. According to epidemiological studies, the prevalence of infections caused by DEC varies in different geographical regions. In study of Orni et al. on 2629 E.coli samples from children under 12 years of age, $16.9 \%$ of the samples were identified as DEC (14). In a study in Vietnam, the prevalence of DEC pathogens was examined and the highest prevalence $(27.5 \%)$ was related to Shigella and E. coli (15). In a study in Bangladesh, Albert et al. examined diarrheal and non-diarrheal stool samples collected from children and found that the most common serotypes were O125, O127, O114, O126 O55, O128, O142, and O119, which were related to EPEC (16). In the present study, the most frequently isolated serotypes were O127 O126, O128, O119, O55, O111, and O86. In line with our findings, phenotypic examination of DEC stains in study of Al-Gallas et al. showed that the most common serotypes were associated with O86, O111, O128 O127, O55, O125, and O126, respectively (17). In a study conducted by Turhanoglu et al., most DEC strains from children with diarrhea were related to serotypes O111: K58, O55: K59, O127: K63, O26: K60, O124: K72, and O125: K70 [18]. In a study conducted by Adugna et al. on 204 E. coli isolates from children aged under five years, $39.2 \%$ of strains have to do with the serotypes O26:K60, O55:K59, O111:K58, O119:K69, and O126:K71 (19). Antibiotic resistance pattern of $E$. coli species varies widely among different countries (20). Majority of serotypes identified in study of Chandaran et al. were connected to the serotypes O25, O101, O22, and O33, and most bacteria were resistance against vancomycin and novobiocin (21).The Adugna study conducted on 422 children with diarrhea indicated that $E$. coli isolates showed high resistance rate against ampicillin, tetracycline and cotrimoxzole than to the drugs commonly used to treat diarrhea caused by $E$. coli, including ampicillin, cotrimoxazole. (22). In a study conducted by Zarrin Ghalam Moghaddam et al. in Tabriz, most serotypes reacted with antiserum 3 (23). However, in our study, most identified serotypes reacted with antiserum 2. In a study conducted by Haggi et al. on children aged less than five years, most isolated were ETEC and resistant to erythromycin and azithromycin but sensitive to imipenem (24). In a similar study on antibiotic resistance pattern in DEC strains, the highest rate of antibiotic resistance was related to ampicillin and co-trimoxazole, while the lowest rate of resistance was observed against ciprofloxacin (25). Aslani reported that majority of DEC strains are resistant to tetracycline, ampicillin, amoxicillin, clavulanic acid, and trimethoprim (26). Similar to most previous studies, we found that the highest rate of antibiotic resistance was related to ampicillin, tetracycline, and co-trimoxazole. The high rate of resistance to tetracycline in children with diarrhea might indicate improper use of this antibiotic. Since tetracycline is cheap antibiotic with few side effects, it is widely used for the treatment of human and animal infections. Excessive and uncontrolled use of this antibiotic can increase emergence of resistant strains (27). The lowest rate of antibiotic resistance was related to imipenem and ciprofloxacin. Ciprofloxacin is used for 
the treatment of intestinal infections caused by E. coli. However, since both imipenem and ciprofloxacin cannot be used for children, cefixime is recommended for treatment of diarrhea caused by DEC strains.

\section{CONCLUSION}

DEC pathotypes are important causes of diarrhea among children admitted to hospitals of Zahedan. Regarding the high rate of antibiotic resistance among these pathotypes in this region, physicians try to prescribe antibiotics based on accurate detection of these

\section{REFERENCES}

1. Jafari A, Aslani MM, Bouzari S. Escherichia coli: a brief review of diarrheagenic pathotypes and their role in diarrheal diseases in Iran. Iranian journal of microbiology. 2012; 4(3): 102-117.

2. Schultsz C, van den Ende J, Cobelens F, Vervoort T, van Gompel A, Wetsteyn JC, et al. Diarrheagenic Escherichia coli and acute and persistent diarrhea in returned travelers. Journal of clinical microbiology. 2000; 38(10): 3550-3554.

3.Bueris V, Sircili MP, Taddei CR, aurilioernandes dos Santos MF, arcia egina Franzolin MR, arina aquerizo Martinez MB, et al. Detection of diarrheagenic Escherichia coli from children with and without diarrhea in Salvador, Bahia, Brazil. Memorias do Instituto Oswaldo Cruz. 2007; 102(7): 839-844.

4. Vidal M, Kruger E, Durán C, Lagos R, Levine M, Prado V, et al. Single multiplex PCR assay to identify simultaneously the six categories of diarrheagenic Escherichia coli associated with enteric infections. Journal of clinical microbiology. 2005; 43(10): 53625365 .

5. Sabaté M, Prats G, Moreno E, Ballesté E, Blanch AR, Andreu A. Virulence and antimicrobial resistance profiles among Escherichia coli strains isolated from human and animal wastewater. Research in Microbiology, 2008; 159(4): 288-93. doi: 10.1016/j.resmic.2008.02.001.

6. Kumarasinghe G, Lim YS, Chow C, Bassett DC. Prevalence of bacterial agents of diarrhoeal disease at the National University Hospital, Singapore and their resistance to antimicrobial agents. Tropical and geographical medicine. 1992; 44(3): 229-232.

7. Aranda KR, Fagundes-Neto U, Scaletsky IC. Evaluation of multiplex PCRs for diagnosis of infection with diarrheagenic Escherichia coli and Shigella spp. Journal of Clinical Microbiology. 2004; 42(12): 58495853.

8.Churgay CA, Aftab Z. Gastroenteritis in children: part I. diagnosis. American family physician. 2012; 85(11): 1059.

9.O'Ryan M, Prado V, Pickering LK. A millennium update on pediatric diarrheal illness in the developing world. in Seminars in pediatric infectious diseases. Elsevier. 2005. strains.

\section{ACKNOWLEDGEMENTS}

The present article is part of a Master's thesis supported by the Zahedan University of Medical Sciences. The authors would like to thank the Deputy of Research and its personnel for their cooperation.

\section{CONFLICT OF INTEREST}

All contributing authors declare no conflict of interest.

10.Feizizadeh S, Salehi-Abargouei A, Akbari V. Efficacy and safety of Saccharomyces boulardii for acute diarrhea. Pediatrics. 2014; 134(1): e176-91. doi: 10.1542/peds.2013-3950.

11. Kaper JB, Nataro JP, Mobley HL. Mobley, Pathogenic escherichia coli. Nature reviews. Microbiology. 2004; 2(2): 123-40.

12. Clarke RC, Wilson JB, Read SC. Growing concerns and recent outbreaks involving non-O157: $H 7$ serotypes of verotoxigenic Escherichia coli. Journal of Food Protection. 1996; 59(10): 1112-1122.

13. Denno DM, Shaikh N, Stapp JR, Qin X, Hutter CM, Hoffman V, et al. Diarrhea etiology in a pediatric emergency department: a case control study. Clinical infectious diseases. 2012; 55(7): 897-904.

14. Yavzori M, Porath N, Ochana O, Dagan R, OrniWasserlauf R, Cohen D, et al. Detection of enterotoxigenic Escherichia coli in stool specimens by polymerase chain reaction. Diagnostic microbiology and infectious disease. 1998; 31(4): 503-509.

15. Hien BT, Scheutz F, Cam PD, Serichantalergs O, Huong TT, Thu TM, Dalsgaard A. Diarrheagenic Escherichia coli and Shigella strains isolated from children in a hospital case-control study in Hanoi, Vietnam. J Clin Microbiol. 2008; 46(3):996-1004. doi: 10.1128/JCM.01219-07.

16. Albert MJ, Faruque SM, Faruque AS, Neogi PK, Ansaruzzaman M, Bhuiyan NA, et al. Controlled study of Escherichia coli diarrheal infections in Bangladeshi children. Journal of Clinical Microbiology. 1995; 33(4): 973-977.

17. -Gallas N, Bahri O, Bouratbeen A, Ben Haasen A, Ben Aissa R. Etiology of acute diarrhea in children and adults in Tunis, Tunisia, with emphasis on diarrheagenic Escherichia coli: prevalence, phenotyping, and molecular epidemiology. The American journal of tropical medicine and hygiene. 2007; 77(3): 571-582.

18.Turhanoglu M. The frequency of Escherichia coli (EPEC, ETEC, EIEC and serotypes) shigella, rotavirus and parasite agents among children with acute gastroenteritis in Southeast Anatolia, Turkey. African Journal of Microbiology Research. 2012; 6(23): 50205024. 
19. Adugna A, Kibret M, Abera B, Nibret E, Adal M. Antibiogram of $E$. coli serotypes isolated from children aged under five with acute diarrhea in Bahir Dar town. African health sciences. 2015; 15(2): 656-664.

20. Dethlefsen L, McFall-Ngai M, Relman DA. An ecological and evolutionary perspective on humanmicrobe mutualism and disease. Nature. 2007; 449(7164): 811-8.

21. Chandran A, Hatha AA, Varghese S, Sheeja KM. Prevalence of multiple drug resistant Escherichia coli serotypes in a tropical estuary, India. Microbes and environments. 2008; 23(2): 153-158.

22. Adugna A, Kibret M, Abera B, Nibret E, Adal M. Antibiogram of $E$. coli serotypes isolated from children aged under five with acute diarrhea in Bahir Dar town. Afr Health Sci. 2015; 15(2): 656-664.

23. Goudarzi H , Zarringhalam M , Nahaei MR , AngoutiG , Safaeyan F. Detection of Escherishia coli pathotypes and their antibiotic resistance in cases of diarrhea in hospitals of Tabriz in 2013. Research in Medicine, 2015; 38(4): 233-238.
24. Haghi F, Zeighami H, Hajiahmadi F, Khoshvaght H, Bayat M. Frequency and antimicrobial resistance of diarrhoeagenic Escherichia coli from young children in Iran. Journal of medical microbiology. 2014; 63(Pt 3): 427-32. doi: 10.1099/jmm.0.064600-0.

25. Alikhani MY, Hashemi SH, Aslani MM, Farajnia S. Prevalence and antibiotic resistance patterns of diarrheagenic Escherichia coli isolated from adolescents and adults in Hamedan, Western Iran. Iranian journal of microbiology. 2013; 5(1): 42-7.

26. Aslani MM, Ahrabi SS, Alikhani YM, Jafari F, Zali RM, Mani M. Molecular detection and antimicrobial resistance of diarrheagenic Escherichia coli strains isolated from diarrheal cases. Saudi medical journal. 2008; 29(3): 388-92.

27.Chopra I, Roberts M. Tetracycline antibiotics: mode of action, applications, molecular biology, and epidemiology of bacterial resistance. Microbiology and molecular biology reviews. 2001; 65(2): 232-260. 